\title{
Unifying Inflation and Dark Matter with Neutrino Masses
}

\author{
Rouzbeh Allahverdi, ${ }^{1,2}$ Bhaskar Dutta, ${ }^{3}$ and Anupam Mazumdar ${ }^{4,5}$ \\ ${ }^{1}$ Perimeter Institute for Theoretical Physics, Waterloo, Ontario, N2L 2Y5, Canada \\ ${ }^{2}$ Department of Physics and Astronomy, University of New Mexico, Albuquerque, New Mexico 87131, USA \\ ${ }^{3}$ Department of Physics, Texas A\&M University, College Station, Texas 77843-4242, USA \\ ${ }^{4}$ NORDITA and Niels Bohr Institute, Blegdamsvej-17, Copenhagen-2100, Denmark \\ ${ }^{5}$ Physics Department, Lancaster University, Lancaster, LA1 4YB, United Kingdom
}

(Received 11 September 2007; published 28 December 2007)

\begin{abstract}
We propose a simple model where a gauge-invariant inflaton is responsible for cosmic inflation and generates the seed for structure formation, while its relic thermal abundance explains the missing matter of the Universe in the form of cold dark matter. The inflaton self-coupling also explains the observed neutrino masses. All the virtues can be attained in a minimal extension of the standard model gauge group around the $\mathrm{TeV}$ scale. We can also unveil these properties of an inflaton in forthcoming space and ground based experiments.
\end{abstract}

DOI: 10.1103/PhysRevLett.99.261301

There are three important puzzles, the origin of inflation, the origin of cold dark matter, and the origin of neutrino masses, which require explanation in any extension of the electroweak standard model (SM). The aim of this Letter is to bind the three issues together and explain all of them in a single setup. Inflation is driven by a scalar field known as the inflaton; for a review, see [1]. In this Letter we shall illustrate an inflaton part of which directly decays into the SM baryons, its thermal relic abundance accounts for the missing matter in the Universe, commonly known as cold dark matter, and also explains the observed neutrino masses.

The inflaton potential explains the flatness of the Universe and also the observed anisotropy in the cosmic microwave background temperature, i.e. $\delta_{H} \sim 1.91 \times$ $10^{-5}$ with an observed range of spectral index: $0.92 \leq$ $n_{s} \leq 1.0$ (within $2 \sigma$ error bar) [2]. The inflaton carries SM gauge charges, and hence naturally produces (a thermal bath of) the SM degrees of freedom $[3,4]$. If the reheat temperature of the Universe is higher than the mass of the inflaton, then the plasma upon reheating will, in addition, have a thermal distribution of the inflaton quanta. If the inflaton is absolutely stable, due to some symmetry, then it can also serve as the cold dark matter. Once the temperature drops below the inflaton mass, its quanta undergo thermal freeze-out and may yield the correct dark matter abundance. As a consequence, direct and indirect dark matter detection experiments will provide valuable information about the inflaton couplings to the SM fields.

The main question is whether relic density of the inflaton after thermal freeze-out can account for dark matter in the Universe. For particles with gauge interactions, the unitarity bound puts an absolute upper bound $\sim 100 \mathrm{TeV}$ on their mass [5]. For smaller couplings this bound will decrease in order to have acceptable thermal dark matter; for a review, see [6]. Therefore, the inflaton mass should be below $100 \mathrm{TeV}$, which puts a severe constraint on the flatness of the inflaton potential. As we will show, an inflaton with a
PACS numbers: 98.80.Cq, 12.60.Jv, 14.60.Pq, 95.35.+d

weak scale mass and a tiny self-coupling can drive a successful inflation. Coincidentally such a small coupling is welcoming if the neutrinos are Dirac in nature [7]. It turns out that if the inflaton is composed of the standard model Higgs boson, slepton, and the sneutrino, then it serves all three purposes: inflation, cold dark matter, and neutrino mass.

Let us consider the minimal supersymmetric standard model (MSSM) with three right-handed (RH) neutrino multiplets. We assume the neutrinos to be of Dirac type. Whether the nature of neutrino is Dirac or Majorana can be determined in the future neutrinoless double beta decay experiment. Then the relevant superpotential term is

$$
W \supset h \mathbf{N H}_{u} \mathbf{L} .
$$

Here $\mathbf{N}, \mathbf{L}$, and $\mathbf{H}_{u}$ are superfields containing the RH neutrinos, left-handed (LH) leptons, and the Higgs boson which gives mass to the up-type quarks, respectively. For conciseness we have omitted the generation indices, and we work in a basis where neutrino Yukawa couplings $h$ (and hence neutrino masses) are diagonalized.

For various reasons, which will become clear, the inclusion of a gauge symmetry under which the RH (s)neutrinos are not singlet is crucial. As far as inflation is concerned, a singlet RH sneutrino would not form a gauge-invariant inflaton along with the Higgs boson and slepton fields. We consider a minimal extension of the SM gauge group which includes a $U(1): S U(3)_{c} \times S U(2)_{L} \times$ $U(1)_{Y} \times U(1)_{B-L}$, where $B$ and $L$ denote the baryon and lepton numbers, respectively. This is the simplest extension of the SM symmetry which is also well motivated: anomaly cancellation requires that three RH neutrinos exist, so that they pair with LH neutrinos to form three Dirac fermions.

In this model we have an extra $Z$ boson $\left(Z^{\prime}\right)$ and one extra gaugino $\left(\tilde{Z}^{\prime}\right)$. The $U(1)_{B-L}$ gets broken around $\mathrm{TeV}$ by new Higgs boson fields with $B-L= \pm 1$. This also prohibits a Majorana mass for the $\mathrm{RH}$ neutrinos at the renormalizable level (note that $\mathbf{N N}$ has $B-L=2$ ). The 
Majorana mass can be induced by a nonrenormalizable operator, but it will be very small.

The value of $h$ needs to be small, i.e. $h \lessgtr 10^{-12}$, in order to explain the light neutrino mass, $\sim \mathcal{O}(0.1 \mathrm{eV})$, corresponding to the atmospheric neutrino oscillations detected by Super-Kamiokande experiment. (One can assume that the neutrino mass terms are part of the Kähler potential (Ref. [8]). One can then generate a small neutrino mass in the superpotential by Kähler transformation (similar to generation of $\mu$ parameter).

Note that the $\mathbf{N H}_{u} \mathbf{L}$ monomial represents a $D$-flat direction under the $U(1)_{B-L}$, as well as the SM gauge group [9]. (A flat direction represented by a polynomial is more involved [10].) The flat direction field $\phi$ is defined as

$$
\phi=\frac{\tilde{N}+H_{u}+\tilde{L}}{\sqrt{3}},
$$

where $\tilde{N}, \tilde{L}, H_{u}$ are the scalar components of corresponding superfields. Since the RH sneutrino $\tilde{N}$ is a singlet under the SM gauge group, its mass receives the smallest contribution from quantum corrections due to SM gauge interactions, and hence it can be set to be the lightest supersymmetric particle (LSP). Therefore the dark matter candidate arises from the RH sneutrino component of the inflaton; see Eq. (2). (RH sneutrino has been considered as dark matter candidate in other contexts [11,12].) The potential along the flat direction, after the minimization along the angular direction, is found to be [4],

$$
V(|\phi|)=\frac{m_{\phi}^{2}}{2}|\phi|^{2}+\frac{h^{2}}{12}|\phi|^{4}-\frac{A h}{6 \sqrt{3}}|\phi|^{3} .
$$

The importance of $A$ term in providing large vacuum energy was shown before [13]. The flat direction mass $m_{\phi}$ is given in terms of $\tilde{N}, H_{u}, \tilde{L}$ masses:

$$
m_{\phi}^{2}=\frac{m_{\tilde{N}}^{2}+m_{H_{u}}^{2}+m_{\tilde{L}}^{2}}{3} .
$$

Note that for $A=4 m_{\phi}$, there exists a saddle point for which $V^{\prime}\left(\phi_{0}\right)=V^{\prime \prime}\left(\phi_{0}\right)=0$. The saddle point and the potential are given by

$$
\begin{gathered}
\phi_{0}=\sqrt{3} \frac{m_{\phi}}{h}=6 \times 10^{12} m_{\phi}\left(\frac{0.05 \mathrm{eV}}{m_{\nu}}\right), \\
V\left(\phi_{0}\right)=\frac{m_{\phi}^{4}}{4 h^{2}}=3 \times 10^{24} m_{\phi}^{4}\left(\frac{0.05 \mathrm{eV}}{m_{\nu}}\right)^{2} .
\end{gathered}
$$

Here $m_{\nu}$ denotes the neutrino mass which is given by $m_{\nu}=$ $h\left\langle H_{u}\right\rangle$, with $\left\langle H_{u}\right\rangle \simeq 174 \mathrm{GeV}$. For neutrino masses with a hierarchical pattern, the largest neutrino mass is $m_{\nu} \simeq$ $0.05 \mathrm{eV}$ in order to explain the atmospheric neutrino oscillations [14], while the current upper bound on the sum of the neutrino masses from cosmology, using Wilkinson Microwave Anisotropy Probe (WMAP) and Sloan Digital Sky Survey (SDSS) data alone, is $0.94 \mathrm{eV} \mathrm{[15].}$

Inflation can take place along the gauge-invariant flat direction $\phi$, near a saddle point $\phi_{0}$. (This requires that the inflaton starts in the vicinity of $\phi_{0}$ with $\dot{\phi}_{0} \approx 0$ [16]. Around a saddle point there exists a self-reproduction regime such that most parts of the Universe inflate forever [4].) The dynamics of the inflaton can be understood by expanding the potential, $V(\phi)=V\left(\phi_{0}\right)+$ $(1 / 3 !) V^{\prime \prime \prime}\left(\phi_{0}\right)\left(\phi-\phi_{0}\right)^{3}+\cdots$, see Refs. [3,4]. The slow roll inflation takes place within an interval $\Delta \phi \sim \phi_{0}^{3} / M_{P}^{2}$ in the vicinity of $\phi_{0}$, and is governed by the third derivative of the potential, $V^{\prime \prime \prime}\left(\phi_{0}\right)=(2 / \sqrt{3}) h m_{\phi}[3,17]$. A sufficient number of $e$ foldings is generated during the slow roll, i.e., $\mathcal{N}_{e} \sim\left(\phi_{0}^{2} / m_{\phi} M_{P}\right)^{1 / 2}$ [4]. For $m_{\phi} \sim 50 \mathrm{GeV}$, it turns out to be $\mathcal{N}_{e} \sim 10^{3}$.

The amplitude of density perturbations follows [4].

$$
\delta_{H} \simeq \frac{1}{5 \pi} \frac{H_{\mathrm{inf}}^{2}}{\dot{\phi}} \simeq 3.5 \times 10^{-27}\left(\frac{m_{\nu}}{0.05 \mathrm{eV}}\right)^{2}\left(\frac{M_{P}}{m_{\phi}}\right) \mathcal{N}_{\mathrm{COBE}}^{2}
$$

where $H_{\text {inf }}$ denotes the Hubble rate during inflation which is given by $H_{\mathrm{inf}}^{2}=8 V\left(\phi_{0}\right) / 3 M_{P}^{2}$. Here $m_{\phi}$ denotes the loop-corrected value of the inflaton mass at the scale $\phi_{0}$ in Eqs. (5)-(7). (Even though the inflaton potential gets loop corrections due to gauge interactions, but the existence of a saddle point remains and the flatness of the potential is not ruined [4].) In Fig. 1, we show the neutrino mass as a function of the inflaton mass. We use $\delta_{H}=$ $1.91 \times 10^{-5}$ to draw the plot. We see that the neutrino mass in the range $0-0.30 \mathrm{eV}$ corresponds to the inflaton mass of $0-30 \mathrm{GeV}$. We will be using this mass range of the inflaton to calculate the supersymmetry (SUSY) masses which allow a RH sneutrino dark matter. It is interesting to note that $m_{\phi} \geq 10^{3} H_{\text {inf }}$ in this mass range; see Eq. (6). This implies that supergravity (SUGRA) corrections to the inflaton potential are negligible. (For an explicit realization of inflation near a saddle point in supergravity, see [18].)

The spectral index of the power spectrum is given by $n_{s}=1+2 \eta-6 \epsilon \simeq 1-4 / \mathcal{N}_{\mathrm{COBE}}$, and the running in the spectral tilt is $d n_{s} / d \ln k=-4 / \mathcal{N}_{\text {COBE }}^{2}$ [4]. Here $\mathcal{N}_{\text {COBE }}$ is the number of $e$ foldings between the time the observationally relevant perturbations were generated and the end of inflation. The exact number depends on the scale of inflation and on when the Universe becomes radiation dominated. As we shall see that the Universe becomes

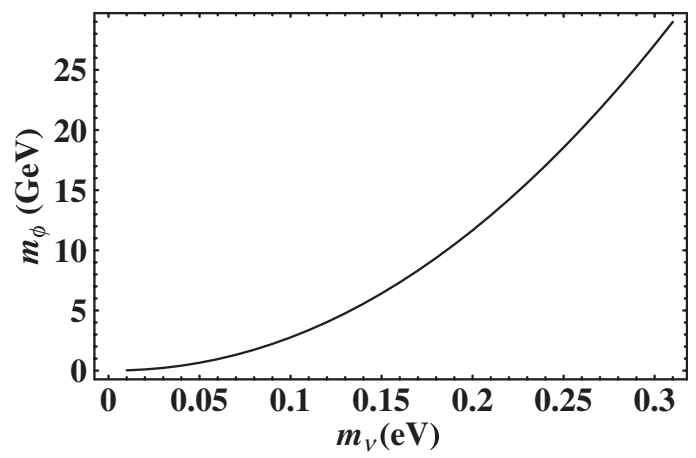

FIG. 1. The inflaton mass $m_{\phi}$ is plotted as a function of the neutrino mass $m_{\nu}$. 
radiation dominated instantly (time scale comparable to the Hubble scale) after the end of inflation. The required number of $e$ foldings is rather small, i.e., $\mathcal{N}_{\mathrm{COBE}} \sim 40$ [19]. For such a low $\mathcal{N}_{\mathrm{COBE}}$ the value of $n_{s} \sim 0.90$ near the saddle point. At a face value this is already ruled out by the WMAP data [2]. However, the spectral tilt can be obtained within $0.90 \leq n_{s} \leq 1.0$ if $\phi_{0}$ becomes a point of inflection, i.e., $V^{\prime}\left(\phi_{0}\right) \neq 0, V^{\prime \prime}\left(\phi_{0}\right)=0[17,20]$. This happens for a slight departure from a saddle point $\left(\mid A^{2}-\right.$ $\left.\left.16 m_{\phi}^{2}\right|^{1 / 2} / 4 m_{\phi}\right) \lesssim 10^{-8}$ [17]. Note that its reflection on the amplitude of the density perturbations is negligible.

The inflaton has gauge couplings to the electroweak and $U(1)_{B-L}$ gauge or gaugino fields. It therefore induces a vacuum expectation value (VEV)-dependent mass $\sim g\langle\phi\rangle$ for these fields ( $g$ denotes a typical gauge coupling). After the end of inflation, $\phi$ starts oscillating around the global minimum at the origin with a frequency $m_{\phi} \sim 10^{3} H_{\text {inf }}$. When the inflaton passes through the minimum, $\langle\phi\rangle=0$, the induced mass undergoes nonadiabatic time variation. This results in nonperturbative particle production [21]. As the inflaton VEV is rolling back to its maximum value $\phi_{0}$, the mass of the gauge or gaugino quanta increases again. Because of their large couplings they quickly decay to the fields which are not coupled to the inflaton, hence massless, notably the down-type (s)quarks. This is a very efficient process as a result of which relativistic particles are created within few Hubble times after the end of inflation (for more details, see [17]). A thermal bath of MSSM particles is eventually formed with a temperature $T_{R} \sim$ $10^{6} \mathrm{GeV}$ (for details of thermalization in SUSY, see [22]). Note that the reheat temperature is high enough for the (hot or cold) electroweak baryogenesis [23]. On the other hand, it is sufficiently low to avoid overproduction of dangerous relics such as gravitinos [22,24].

Scatterings via the new $U(1)$ gauge interactions also bring the RH sneutrino into thermal equilibrium. Note that part of the inflaton, i.e., its $\tilde{N}$ component, see Eq. (2), has never decayed; only the coherence in the original condensate that drives inflation is lost. Its relic abundance will then be set by thermal freeze-out. The fact that $\tilde{N}$ has gauge interactions under the new $U(1)$ is crucial in this respect: the neutrino Yukawa $h$ is way too small to allow acceptable thermal dark matter. (Or acceptable sneutrino dark matter at all. Note that $\tilde{N}$ would dominate the Universe right after the end of inflation if it had no gauge interactions since $h$ is too small to deplete the energy in the $\tilde{N}$ component of the inflaton via nonperturbative particle production.)

Note that the mass of dark matter (i.e., RH sneutrino) is correlated with the inflaton mass; see Eq. (4). However, the former is calculated at the weak scale, while the latter is at the scale $\phi_{0}$; see Eq. (5). The two quantities are related to each other by renormalization group equations (RGEs). In order to calculate the masses in the model, we use SUGRA boundary conditions [i.e., $m_{0}$, universal scalar mass for the squarks and sleptons (but the Higgs boson masses are different); $m_{1 / 2}$, universal gaugino mass; $A_{0}$, trilinear sca- lar coupling, $\mu>0$ and $\tan \beta$ ] for the sparticle masses in the extended $U(1)$ model. We also assume that the gauge couplings are unified at the grand unified theory scale. Then the new gauge coupling is of the order of hypercharge gauge coupling.

Even though the inflaton mass is small at the scale $\phi_{0}$, obtained by solving Eq. (5), the RGE effects increase the RH sneutrino and slepton masses at the weak scale. Note that $m_{\tilde{N}}$ and $m_{\tilde{L}}$ are soft breaking masses, while $m_{H_{u}}$ also includes the contribution from the $\mu$ term. In Fig. 2 we plot the RH sneutrino and top square masses at the weak scale for different values of gluino masses. The gluino masses for the three lines (solid lines and dashed lines) are $730 \mathrm{GeV}, 1.20 \mathrm{TeV}$, and $1.64 \mathrm{TeV}$ (from the bottom to the top). The lines are drawn for $0.30 \mathrm{eV}$ neutrino mass. If we choose the neutrino masses to be $0.05 \mathrm{eV}$, the lines do not shift much. The left and right end of the lines correspond to $m_{0}=0$ and $m_{\tilde{\nu}}>m_{\tilde{\chi}}$. The charged sleptons and the $\mathrm{LH}$ sneutrinos are more massive compared to the $\mathrm{RH}$ sneutrino since these particles get contributions from more gaugino loops due to their $S U(2)_{L}$ and $U(1)_{Y}$ couplings. Figure 2 shows that the masses of sparticles (e.g., the lighter top squark) can be within the reach of initial phase of the LHC.

In order to calculate the relic abundance of the $\mathrm{RH}$ sneutrino, we need to know the masses of the additional gauge boson $Z^{\prime}$ and its SUSY partner $\tilde{Z}^{\prime}$, the new Higgsino masses, Higgs VEVs which break the new $U(1)$ gauge symmetry, the RH sneutrino mass, the new gauge coupling, and the charge assignments for the additional $U(1)$. We assume that the new gauge symmetry is broken around $2 \mathrm{TeV}$ (this is sufficient to ensure that the RH neutrinos decouple from the thermal bath early enough in order not to affect big bang nucleosynthesis), and the existence of two new Higgs boson superfields to maintain the theory anomaly free. The primary diagrams responsible to provide the right amount of relic density are mediated by $\tilde{Z}^{\prime}$ in the $t$ channel. In Fig. 3, we show the relic density values for smaller masses of sneutrino where the lighter top squark mass is $\lesssim 1 \mathrm{TeV}$. The smaller top squark mass will be

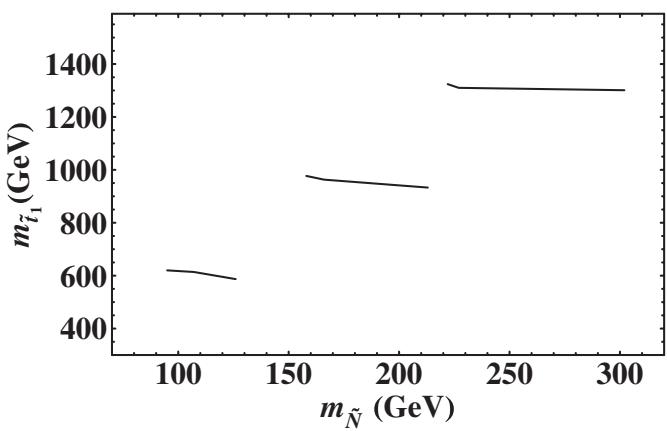

FIG. 2. $m_{\tilde{t}_{1}}$ vs $m_{\tilde{N}}$. The lines are for neutrino masses $0.3 \mathrm{eV}$. The gluino masses for the three lines are 730, 1200 and $1640 \mathrm{GeV}$ (from the bottom to the top). For smaller neutrino masses, the lines are slightly shifted downwards. 


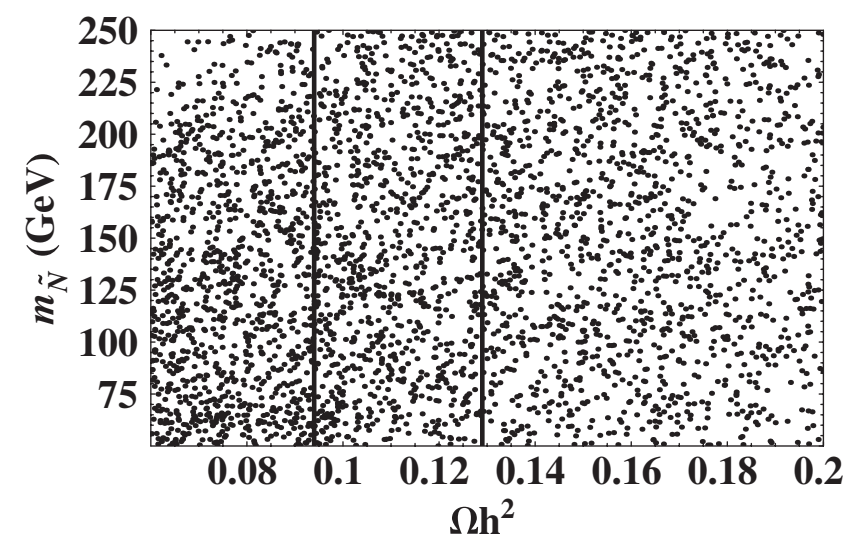

FIG. 3. $\Omega h^{2}$ vs $m_{\tilde{N}}$. The solid lines from left to right are for $\Omega h^{2}=0.094$ and 0.129 , respectively. The $Z^{\prime}$-ino mass is equal to the $B$-ino mass since the new $U(1)$ gauge coupling is the same as the hypercharge gauge coupling.

easily accessible at the LHC and is also preferred by the little hierarchy solutions [25]. We have varied new gaugino and Higgsino masses and the ratio of the VEVs of new Higgs boson fields to generate Fig. 3. We find that the WMAP [2] allowed values of the relic density; i.e., 0.0940.129 is satisfied for many points. In the case of a larger sneutrino mass in this model, the correct dark matter abundance can be obtained by annihilation via $Z^{\prime}$ pole [12].

Since the dark matter candidate, the RH sneutrino, interacts with quarks via the $Z^{\prime}$ boson, it is possible to see it via the direct detection experiments. The detection cross sections are not small as the interaction diagram involves $Z^{\prime}$ in the $t$ channel. The typical cross section is about $2 \times$ $10^{-8} \mathrm{pb}$ for a $Z^{\prime}$ mass around $2 \mathrm{TeV}$. It is possible to probe this model in the upcoming dark matter detection experiments [26]. The signal for this new scenario at the LHC will contain standard jets plus missing energy and jets plus leptons plus missing energy. The jets and the leptons will be produced from the cascade decays of squarks and gluinos into the final state containing the sneutrino. In general, the models with RH sneutrino dark matter will have similar signal as the models with neutralino dark matter. The major difference, however, lies in the fact that the lightest dark matter particle has different spin, i.e., that sneutrino has spin 0 compared to the lightest neutralino whose spin is $1 / 2$. If the spin of LSP can be determined at the LHC, this model can easily be distinguished [27].

We wish to thank Alex Kusenko for helpful discussions. The work of R. A. is supported by Perimeter Institute for Theoretical Physics. B. D. is supported by the U.S. DOE Grant No. DE-FG02-95ER40917. A. M. is partly supported by the UNIVERSE-NET MRTN-CT-2006-035863).

[1] A. Mazumdar, arXiv:0707.3350.

[2] D. N. Spergel et al., Astrophys. J. Suppl. Ser. 170, 377 (2007).
[3] R. Allahverdi et al., Phys. Rev. Lett. 97, 191304 (2006); R. Allahverdi, A. Jokinen, and A. Mazumdar, arXiv:hepph/0610243; R. Allahverdi, B. Dutta, and A. Mazumdar, Phys. Rev. D 75, 075018 (2007).

[4] R. Allahverdi, A. Kusenko, and A. Mazumdar, J. Cosmol. Astropart. Phys. 07 (2007) 018.

[5] K. Griest and M. Kamionkowski, Phys. Rev. Lett. 64, 615 (1990).

[6] G. Bertone, D. Hooper, and J. Silk, Phys. Rep. 405, 279 (2005).

[7] For review, see R. N. Mohapatra et al., Rep. Prog. Phys. 70, 1757 (2007).

[8] R. Arnowitt, B. Dutta, and B. Hu, Nucl. Phys. B682, 347 (2004).

[9] For reviews, see K. Enqvist and A. Mazumdar, Phys. Rep. 380, 99 (2003); M. Dine and A. Kusenko, Rev. Mod. Phys. 76, 1 (2003).

[10] K. Enqvist, A. Jokinen, and A. Mazumdar, J. Cosmol. Astropart. Phys. 01 (2004) 008.

[11] J. McDonald, J. Cosmol. Astropart. Phys. 01 (2007) 001; C. Arina and N. Fornengo, arXiv:0709.4477; T. Asaka, K. Ishiwata, and T. Moroi, Phys. Rev. D 75, 065001 (2007).

[12] H. S. Lee, K. T. Matchev, and S. Nasri, Phys. Rev. D 76, 041302 (2007).

[13] R. Allahverdi et al., J. Cosmol. Astropart. Phys. 10 (2006) 007.

[14] For example, see A. Strumia and F. Vissani, arXiv:hep-ph/ 0606054.

[15] M. Tegmark et al., Phys. Rev. D 74, 123507 (2006).

[16] R. Allahverdi, A. R. Frey, and A. Mazumdar, Phys. Rev. D 76, 026001 (2007).

[17] R. Allahverdi et al., J. Cosmol. Astropart. Phys. 06 (2007) 019.

[18] K. Enqvist, L. Mether, and S. Nurmi, arXiv:0706.2355.

[19] A. R. Liddle and S. M. Leach, Phys. Rev. D 68, 103503 (2003); C. P. Burgess et al., J. High Energy Phys. 05 (2005) 067.

[20] J.C. B. Sanchez, K. Dimopoulos, and D. H. Lyth, J. Cosmol. Astropart. Phys. 01 (2007) 015.

[21] J.H. Traschen and R. H. Brandenberger, Phys. Rev. D 42, 2491 (1990); Y. Shtanov, J. H. Traschen, and R. H. Brandenberger, Phys. Rev. D 51, 5438 (1995); L. Kofman, A.D. Linde, and A. A. Starobinsky, Phys. Rev. Lett. 73, 3195 (1994); Phys. Rev. D 56, 3258 (1997); D. Cormier, K. Heitmann, and A. Mazumdar, Phys. Rev. D 65, 083521 (2002).

[22] R. Allahverdi and A. Mazumdar, arXiv:hep-ph/0505050; J. Cosmol. Astropart. Phys. 10 (2006) 008; Phys. Rev. D 76, 103526 (2007); J. Cosmol. Astropart. Phys. 08 (2007) 023.

[23] V. A. Rubakov and M.E. Shaposhnikov, Usp. Fiz. Nauk 166, 493 (1996) [Phys. Usp. 39, 461 (1996)]; J. GarciaBellido et al., Phys. Rev. D 60, 123504 (1999).

[24] A. L. Maroto and A. Mazumdar, Phys. Rev. Lett. 84, 1655 (2000); G. F. Giudice, A. Riotto, and I. Tkachev, J. High Energy Phys. 11 (1999) 036.

[25] B. Dutta and Y. Mimura, Phys. Lett. B 648, 357 (2007).

[26] L. Baudis, arXiv:0711.3788.

[27] C. Athanasiou et al., J. High Energy Phys. 08 (2006) 055; (to be published). 